\title{
Heterosynaptic Suppression of Developing Neuromuscular Synapses in Culture
}

\author{
Yi-Jiuan Lo and Mu-ming Poo \\ Department of Biological Sciences, Columbia University, New York, New York 10027
}

Activity-dependent competition between two spinal neurons coinnervating an embryonic myocyte was studied in Xenopus cell culture. We have characterized in detail the phenomenon of heterosynaptic suppression by which tetanic stimulation of one neuron results in functional suppression of the synapse made by the untetanized neuron (Lo and Poo, 1991). Fluorescence labeling of the neurons using two different fluorophores revealed that the coinnervating nerve terminals on the spherical myocyte were in close proximity. Heterosynaptic suppression could be induced when the postsynaptic cell was held under either current-clamp or voltage-clamp conditions during the tetanic stimulation. This finding, together with the observation that repetitive postsynaptic depolarization of the myocyte by direct current injection was much less effective in inducing synaptic depression, suggests that postsynaptic ACh receptor activation plays a dominant role in the induction of heterosynaptic suppression. The heterosynaptic suppression appears to be mediated by a rise of $\mathrm{Ca}^{2+}$ levels in the postsynaptic cell, since it was not observed when the cytosolic $\mathrm{Ca}^{2+}$ concentration of the myocyte was buffered at a low level with intracellular loading of a $\mathrm{Ca}^{2+}$ chelator, 1,2-bis(2-aminophenoxy)ethane- $N, N, N^{\prime}, N^{\prime}$-tetra-acetic acid (BAPTA). The dependence of heterosynaptic suppression on the pattern of tetanic stimulation was also studied. At a stimulation frequency of $2 \mathrm{~Hz}$, detectable heterosynaptic suppression could be induced after 20 repetitive stimuli were applied to one of the presynaptic neurons and the suppression was more effective with increasing number of stimuli. Over the range of $0.5-5 \mathrm{~Hz}$, the extent of suppression was independent of the frequency of tetanic stimulation and, in some cells, detectable suppression could be induced at a frequency as low as $0.05 \mathrm{~Hz}$. Except for a few cases, heterosynaptic suppression was found to last for as long as the recording was made after tetanus (up to $1 \mathrm{hr}$ ). The fact that the mean amplitude of spontaneous synaptic currents remained the same before and after the suppression while the evoked synaptic currents exhibited higher fluctuation after suppression suggests that the observed synaptic suppression involves a reduction of evoked $A C h$ release from the nerve terminal, although postsynaptic changes have not been excluded. Fi-

Received May 11, 1993; revised Feb. 7, 1994; accepted Feb. 16, 1994.

This work was supported by grants from National Institute of Health (NS 22764) and National Science Foundation (IBN22106) and Office of Naval Research (N00014-90-J1865). We thank W. B. Gan for help in fluorescence labeling experiments.

Correspondence should be addressed to $\mathrm{Mu}$-ming Poo at the above address. Copyright (c) 1994 Society for Neuroscience $0270-6474 / 94 / 144684-10 \$ 05.00 / 0$ nally, we found that spontaneous synaptic activity may also contribute in part to the synaptic competition between coinnervating nerve terminals. Taken together, these findings provide a quantitative basis for further understanding of activity-dependent competition between developing neuromuscular synapses.

IKey words: synaptic competition, synaptogenesis, synaptic plasticity, neuromuscular junction, cell culture, Xenopus laevis]

Nerve connections in the developing nervous system are susceptible to modulation by electrical activity, a process crucial for the influence of early experience on the development of the nervous system (Purves and Lichtman, 1985; Shatz, 1990). The modulation may involve functional potentiation or suppression of synaptic efficacy as well as stabilization or elimination of nerve connections. One emerging theme of synaptic modulation involves competitive interactions between adjacent synaptic inputs to the same postsynaptic targets (Constantine-Paton et al., 1990). Many aspects of synaptic modulation, for example, the causal relationship between changes in synaptic functions and morphological connectivity, the cellular mechanisms underlying the competitive interaction between synaptic inputs, and the exact time course of activity-dependent synaptic changes, remain largely unknown. Experimental studies on these problems have been hindered by the complexity of nerve connections and the inaccessibility of synaptic sites within the developing nervous tissue. We have recently employed a simple Xenopus nervemuscle culture system to study one aspect of synaptic competition, namely, the activity-dependent functional modulation of coinnervating excitatory nerve terminals on the same postsynaptic target cell (Lo and Poo, 1991). This study showed that developing neuromuscular synapses can be functionally suppressed by repetitive stimulation applied to other neurons coinnervating the same postsynaptic myocyte, while the synaptic efficacy of the stimulated input remains either unchanged or potentiated. In the present study we examine in more detail the proximity of nerve terminals at the myocyte surface, the roles of postsynaptic depolarization, $\mathrm{ACh}$ receptor activation, and postsynaptic $\mathrm{Ca}^{2+}$ influx in the induction of synaptic suppression. Furthermore, we have also studied the effective stimulus parameters and the time course of heterosynaptic suppression. The results from these studies provide further quantitative information concerning activity-dependent synaptic modulation and the underlying cellular mechanısms of this model system.

\section{Materials and Methods}

Culture preparation. Cultures were prepared according to procedures reported previously (Spitzer and Lamborghini, 1976; Anderson et al., 
1977; Tabti and Poo, 1991). Briefly, the neural tube tissue from $1 \mathrm{~d}$ old embryos (stage 20-24, Nieuwkoop and Faber, 1967) was dissociated in $\mathrm{Ca}^{2+} / \mathrm{Mg}^{2+}$-free Ringer's solution supplemented with EDTA, plated on clean glass coverslips, and incubated at room temperature $\left(20-22^{\circ} \mathrm{C}\right)$ for $1 \mathrm{~d}$ prior to the experiment. The culture medium consisted of $50 \%$ $(\mathrm{v} / \mathrm{v})$ Leibovitz medium (GIBCO), $1 \%(\mathrm{v} / \mathrm{v})$ fetal calf serum (GIBCO), and $49 \%(\mathrm{v} / \mathrm{v})$ Ringer's solution (115 $\mathrm{mm} \mathrm{NaCl}, 2 \mathrm{~mm} \mathrm{CaCl}, 2.5 \mathrm{~mm}$ $\mathrm{KCl}$, and $10 \mathrm{~mm}$ HEPES, pH 7.3). Spherical myocytes were used in all experiments. These myocytes showed identical functional characteristics as those of the spindle-shaped or extended myocytes in the same culture (Evers et al., 1989). They were readily innervated by cocultured spinal neurons, and the synapses showed functional and structural features (Buchanan et al., 1989) similar to those of developing neuromuscular synapses in the Xenopus embryo (Kullberg et al., 1977).

Electrophysiology. Recordings of postsynaptic responses were made with the gigaohm-seal whole-cell recording method (Hamill et al., 1981; Evers et al., 1989). Presynaptic extracellular suprathreshold stimulation was applied with heat-polished patch pipettes at the soma under looseseal conditions, following the procedure previously described (Sun and Poo, 1987). Synaptic efficacy was assayed by recording five or six nerveevoked synaptic currents (ESCs) at a low frequency $(0.05 \mathrm{~Hz})$ of stimulation. With the exception of $\mathrm{Ca}^{2+}$ buffering experiments, the solution in the whole-cell recording pipette contained $150 \mathrm{~mm} \mathrm{KCl}, 1 \mathrm{mM} \mathrm{NaCl}$, $1 \mathrm{mM} \mathrm{MgCl}_{2}$, and $10 \mathrm{~mm}$ HEPES (pH 7.2). All recordings were made with a patch-clamp amplifier (EPC 7, List). The data were digitized (Neurodata 384) and stored in a videotape recorder for later playback on an oscillographic recorder (Gould 3200) or for analysis by a microcomputer. Assuming that evoked ACh secretion at these synapses is quantal in nature, we have followed the procedure previously described (Bekkers and Stevens, 1990; Malinow and Tsien, 1990) to analyze the fluctuation of ESC amplitude. The mean ESC amplitude $(m)$ and its variance $(v)$ were measured before and after heterosynaptic suppression was produced. The distribution of the ESC amplitude was assumed to be a binomial distribution modified to take into account the variability of the quantal size. Thus, $m=a \mathrm{~N} p$ and $\nu=a^{2} \mathrm{~N} p(1-p)+a^{2} \mathrm{~N} p$ $\mathrm{c}_{\mathrm{m}}{ }^{2}$, where $a$ is the average quantal size, $\mathrm{N}$ is the total number of a vailable quanta, $p$ is the release probability, and $c_{m}$ is the coefficient of variation of the quanta. The coefficient of variation (CV) is defined as $v^{1 / 2} / m$. The square of $\mathrm{CV}, \mathrm{CV}^{2}=\left(1+\mathrm{c}_{\mathrm{m}}{ }^{2}-p\right) /(\mathrm{N} p)$, is a function independent of the quantal size $a$. If heterosynaptic suppression is due simply to a reduction of postsynaptic sensitivity, then $\mathrm{CV}^{2}$ will not change after suppression, and the ratio of $\mathrm{CV}^{2}$ before and after suppression should remain 1 for all levels of suppression. Substantial deviation from this prediction suggests involvement of presynaptic modulation.

Fluorescence labeling. The presynaptic neurons of a dually innervated spherical myocyte were selectively stained by incorporating fluorescent lipid analogs into the plasma membrane. To incorporate lipid analogs, the cultures were fixed for $30 \mathrm{~min}$ in $4 \%$ paraformaldehyde, followed by extensive washing with Ringer's solution. Glass micropipettes with resistances of about $100 \mathrm{M} \Omega$ were pulled with a microelectrode puller (P-87, Sutter Inst. Co.). The pipettes were then filled with a $1 \%$ solution of either 1,1'-dioctadecyl-3,3,3',3'-tetramethlindocarbocyanine perchlorate (DiI; Molecular Probe, Inc.) or 3,3'-dioctadecyloxacarbocyanine perchlorate (DiO; Molecular Probe, Inc.) in 100\% methylene chloride (Sigma). Under $40 \times$ water immersion optics, the pipette filled with the lipid was brought into contact with the soma of the neuron. Repetitive current pulses $(1 \mathrm{nA}, 500 \mathrm{msec}$, and $1 \mathrm{~Hz})$ were applied to the pipette until a small crystal was formed at the pipette tip. The crystal attached to the surface of the soma and the pipette was removed. The attached lipid crystal was then absorbed by the cell membrane and spread along the entire length of the neuronal surface. Fluorescence images of the neuron were collected with filters specific for DiI and DiO fluorescence and processed into pseudocolors using a confocal microscope (Bio-Rad MRC-600).

\section{Results}

Dual innervation on spherical myocytes

One day following cell plating, many spinal neurons establish functional synaptic contacts with cocultured myocytes. The present study utilized spherical mononucleatcd myocytcs which were innervated by two cocultured spinal neurons. Functional synaptic transmission was monitored by whole-cell, voltageclamp recordings of impulse-evoked synaptic currents (ESCs) of the innervated myocyte. In each experiment, functional innervation by both neurons was first screened by the appearance of ESCs or muscle contraction (in the absence of myocyte recording) in response to extracellular stimulation of either neuronal soma. The use of myocyte contraction as an assay for innervation was convenient, but may not reveal suhthreshold inputs. For a 3 month period we have carried out the screening using only the appearance of ESCs. For all dually innervated myocytes $(n=94), 52 \%$ showed ESCs induced by only one neuron, $20 \%$ showed ESCs induced by both neurons, and the rest showed no detectable ESCs. To ensure that functional connections made by both neurons to the myocyte are monosynaptic in nature, whole-cell recording at the soma of one of the coinnervating neurons was made and the response elicited by stimulating the other neuron was examined. In all cases tested $(n=5)$, we observed no synaptic transmission between the neurons, although we cannot rule the possibility of interactions between the two nerve terminals that escaped our detection.

To examine the morphology of these synapses involving dual innervation, fluorescence labeling of the ncurite was carricd out by incorporating two different fluorescent lipid analogs separately into two neurons that showed functional innervation of the same myocyte. The neuritic contact with the myocyte occurs mostly at the side of the myocyte that is facing the culture substratum. We observed extensive neurite-myocyte contact as well as neurite-neurite contact between innervating neurons. One example of fluorescence labeling is shown in Figure 1. Since the average diameter of the spherical myocyte was $33.5 \pm 8.8$ $\mu \mathrm{m}( \pm \mathrm{SD}, n=20)$, the presynaptic sites between the two innervating neurons examined in the present study must be confined within this range.

\section{Heterosynaptic suppression induced by repetitive presynaptic stimulation}

We have previously found that a single episode of tetanic stimulation of 50-100 pulses applied to one of the two coinnervating neurons resulted in a reduction of the impulse-evoked synaptic response elicited from the untetanized neuron (Lo and Poo, 1991). The tetanus was applied while the myocyte was voltage clamped at the resting myocyte membrane potential. An outstanding question is whether heterosynaptic suppression can be observed when the postsynaptic myocyte is allowed to depolarize during the tetanus and whether postsynaptic depolarization may also contribute to the induction of synaptic suppression. In the present study, we performed a series of experiments in which the whole-cell recording was set at current-clamp condition during the tetanus. Consistent heterosynaptic suppression was observed. Figure 2 illustrates examples of results obtained from $1 \mathrm{~d}$ old cultures, using tetanus under either current-clamp (Fig. $2 a, b$ ) or voltage-clamp (Fig. $2 c$ ) condition, respectively. In all three cases, the average ESCs elicited by the test stimuli showed significant heterosynaptic suppression after 100 tetanic pulses were applied to one of the presynaptic neurons at a frequency of $2 \mathrm{~Hz}$. During the tetanus, the current-clamped myocyte exhibited synaptic potentials and in some cases action potentials as well, while the voltage-clamped myocyte showed ESCs (insets, Fig. 2).

Figure 3, $a$ and $b$, summarizes the results from 31 experiments in which 100 pulses of tetanic stimuli at $2 \mathrm{~Hz}$ were applied to one presynaptic neuron while the myocyte was held in either current-clamp (CC) or voltage-clamp (VC) condition. Changes in the mean ESC amplitude elicited by the tetanized neuron (T) 


\section{a}

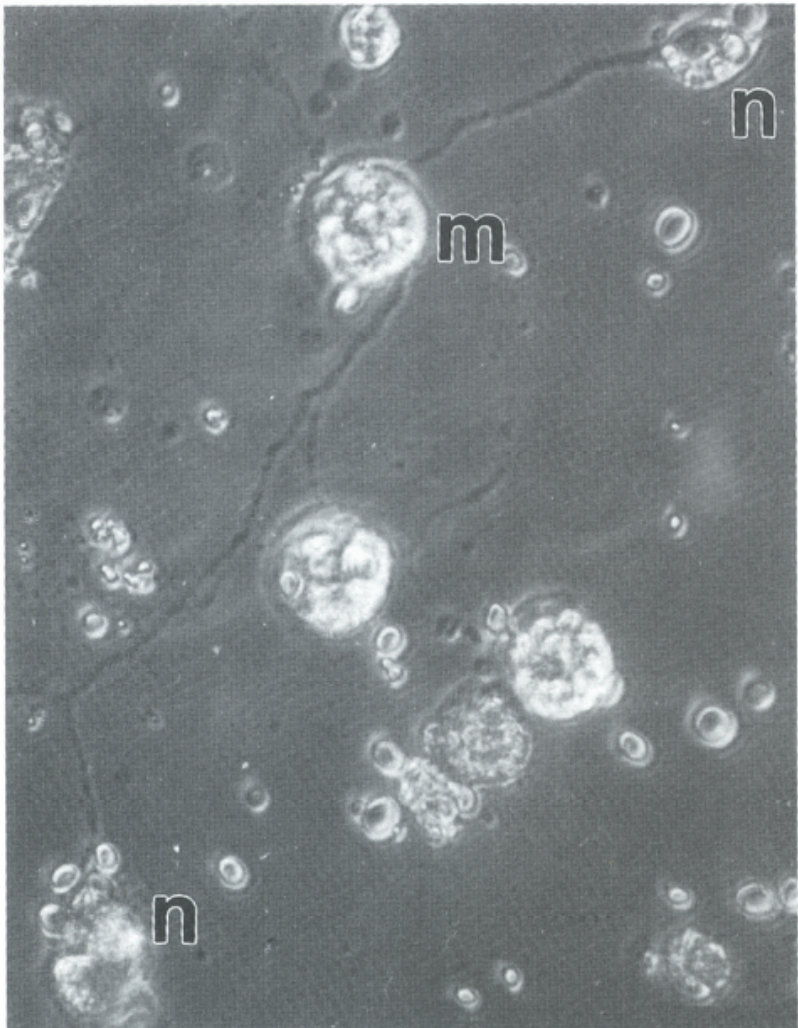

b

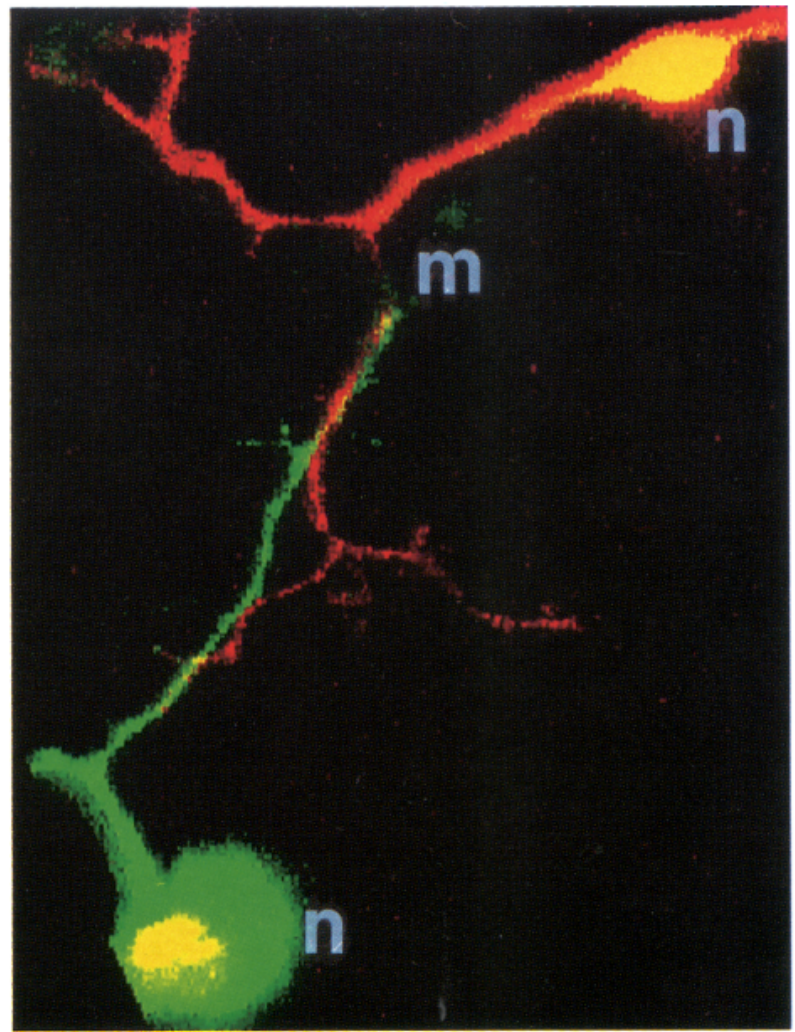

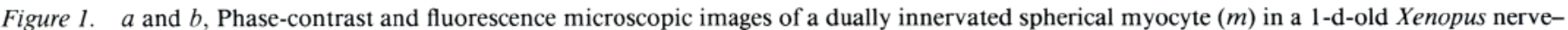

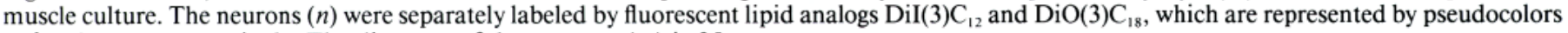
red and green, respectively. The diameter of the myocyte $(m)$ is $35 \mu \mathrm{m}$.

or the untetanized neuron (UT) were calculated for each experiment by normalizing the mean ESC amplitude (within 10 min following the tetanus) against the control mean ESC amplitude (prior to the tetanus). The synaptic response of the tetanized inputs showed no change after the tetanus $(99 \pm 4.9 \%$, \pm SEM, $n=12$, and $106 \pm 6.8 \%, \pm$ SEM, $n=19$, respectively, for $\mathrm{CC}$ and $\mathrm{VC}$ conditions), while that of the untetanized inputs was markedly reduced. The synaptic response after suppression under the CC condition $(24.8 \pm 6.2 \%, \pm$ SEM, $n=12)$ appeared to be slightly lower than that under the VC condition (41.5 \pm $6.7 \%, \pm$ SEM, $n=19 ; p=0.098, t$ test). In the CC condition, all 12 cases showed significant synaptic suppression by heterosynaptic tetanic stimulation ( $p<0.05$, rank sum test). In the VC condition, 4 out of 19 cases showed no significant suppression $(p>0.05$, rank sum test).

\section{The role of postsynaptic depolarization}

Although postsynaptic depolarization during the tetanus was not required for the induction of heterosynaptic suppression (Lo and Poo, 1991), results described above suggest that depolarization may also contribute to the extent of synaptic suppression. This idea was tested directly by examining the effect of repetitive depolarizations of the myocyte membrane in the absence of any synaptic activation. Repetitive suprathreshold depolarizations $(70-100 \mathrm{mV}$ ) were induced (at $2 \mathrm{~Hz}$ for a period of $50 \mathrm{sec}$ ) in a singly innervated myocyte by direct intracellular injection of positive current pulses through a whole-cell recording pipette.
After the depolarizations we observed a small but significant reduction ( $p<0.005, t$ test) in the mean amplitude of ESCs elicited by the innervating neuron $(79.6 \pm 5.8 \%$ of control, $\pm \mathrm{SEM}, n=12$; Fig. 3 c). In comparison to heterosynaptic tetanic stimulation, which resulted in a reduction of mean ESC amplitude to $24.8 \pm 6.2 \%$ and $41.5 \pm 6.7 \%$ of the control, respectively, for CC and VC conditions, repetitive postsynaptic depolarizations by current injections were much less effective in inducing synaptic suppression ( $p<0.005, t$ test). Nevertheless, this small effect produced by depolarizations may account for the slightly higher extent of heterosynaptic suppression observed when the tetanus was applied under current-clamp condition. Extensive studies of the depolarization-induced synaptic changes are reported in the following companion article (Lo et al., 1994).

\section{The role of postsynaptic calcium elevation}

The above results suggest that activation of ACh receptors plays an important role in the induction of heterosynaptic suppression. An immediate consequence of activating ACh receptors in these myocytes is the $\mathrm{Ca}^{2+}$ influx through activated $\mathrm{ACh}$ channels (Decker and Dani, 1990). In the present study, we examined the effect of postsynaptic $\mathrm{Ca}^{2+}$ levels on the induction of heterosynaptic suppression. Calcium concentration was buffered at a low level by adding the $\mathrm{Ca}^{2+}$-buffer BAPTA at a concentration of $5 \mathrm{~mm}$ together with $2 \mathrm{mM} \mathrm{Ca}^{2+}$ to the wholecell pipette. Assuming the $K_{d}$ for BAPTA to be $5 \times 10^{-7} \mathrm{M}$ in 


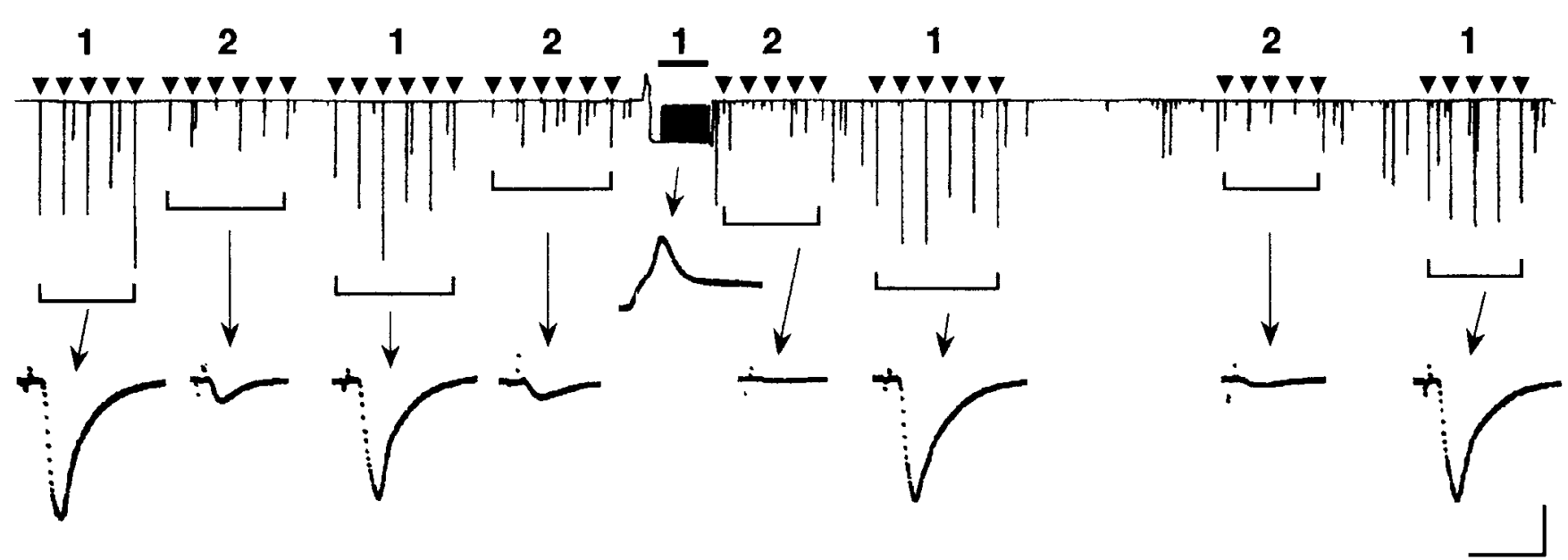

b
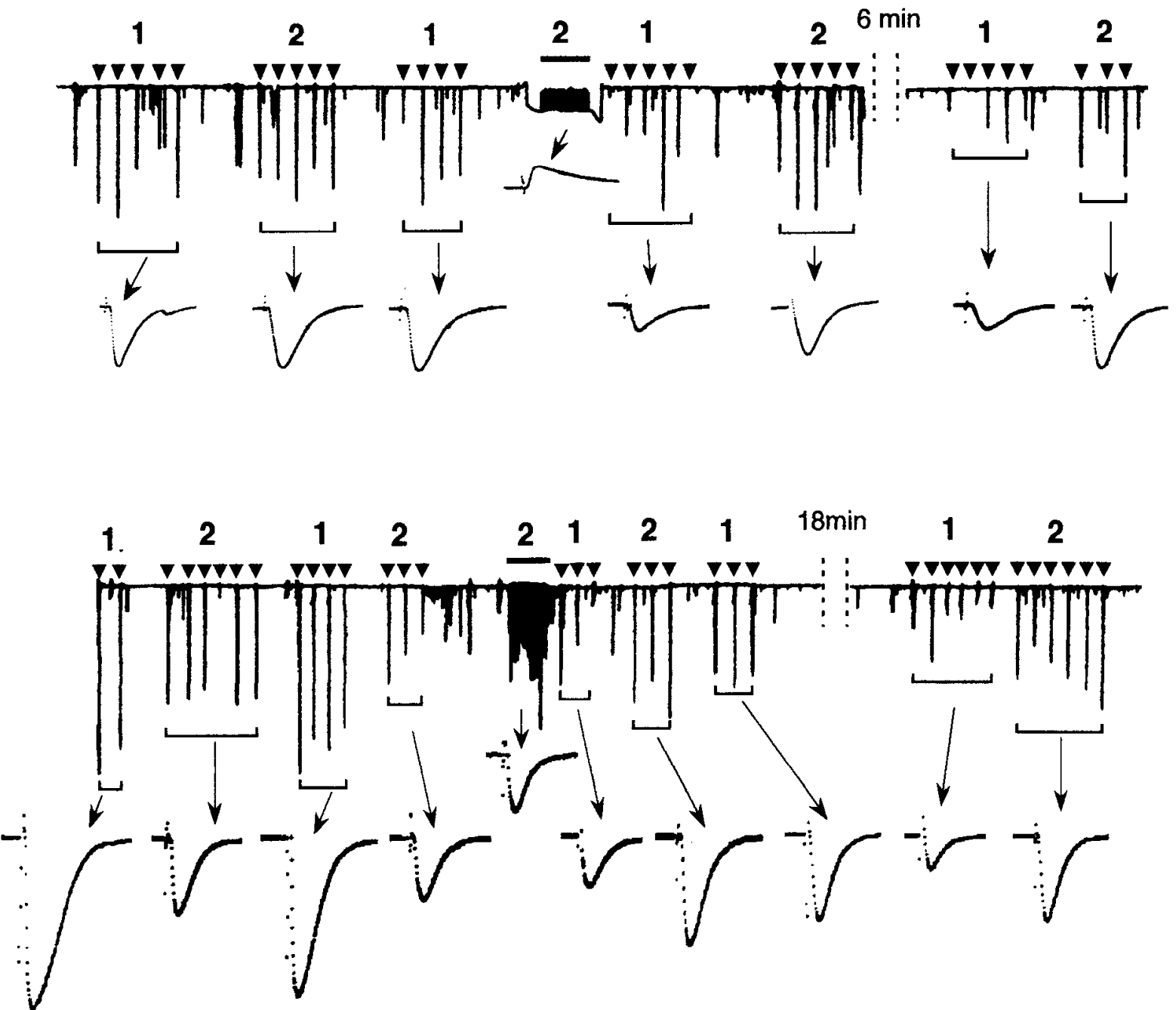

Figure 2. Heterosynaptic suppression of synaptic efficacy. Continuous traces represent the membrane current of a dually innervated myocyte recorded by whole-cell recording method (filtered at $150 \mathrm{~Hz}$ ). Evoked synaptic currents (ESCs) elicited by neuron 1 and 2 marked by triangles were measured alternatively by test stimulation at a low frequency of about $0.05 \mathrm{~Hz}$ and under voltage-clamp condition $(V=-70 \mathrm{mV})$. Calibration: $1 \mathrm{nA}, 1 \mathrm{~min}$. Computer-averaged traces of ESCs and membrane depolarizations during the tetanic stimulation are shown below at higher time resolution (filtered at $2.5 \mathrm{kHz}$ ). Calibration: $a, 1.5 \mathrm{nA}, 15 \mathrm{msec} ; b, 1 \mathrm{nA}, 15 \mathrm{msec} ; c, 1.2 \mathrm{nA}, 10 \mathrm{msec}$. $a$ and $b$, Tetanic stimulation (1wu pulses at $2 \mathrm{~Hz}$ ) was applied under current-clamp conditions to neuron $1(a)$ and $2(b)$ at the time marked by the horizontal bar to induce action potentials (a) and subthreshold synaptic potentials $(b)$. Calibration for depolarization traces: $a ; 50 \mathrm{mV}, 20 \mathrm{msec} ; b, 70 \mathrm{mV}, 30 \mathrm{msec}$. $c$, Tetanus was applied under voltage-clamp condition. 


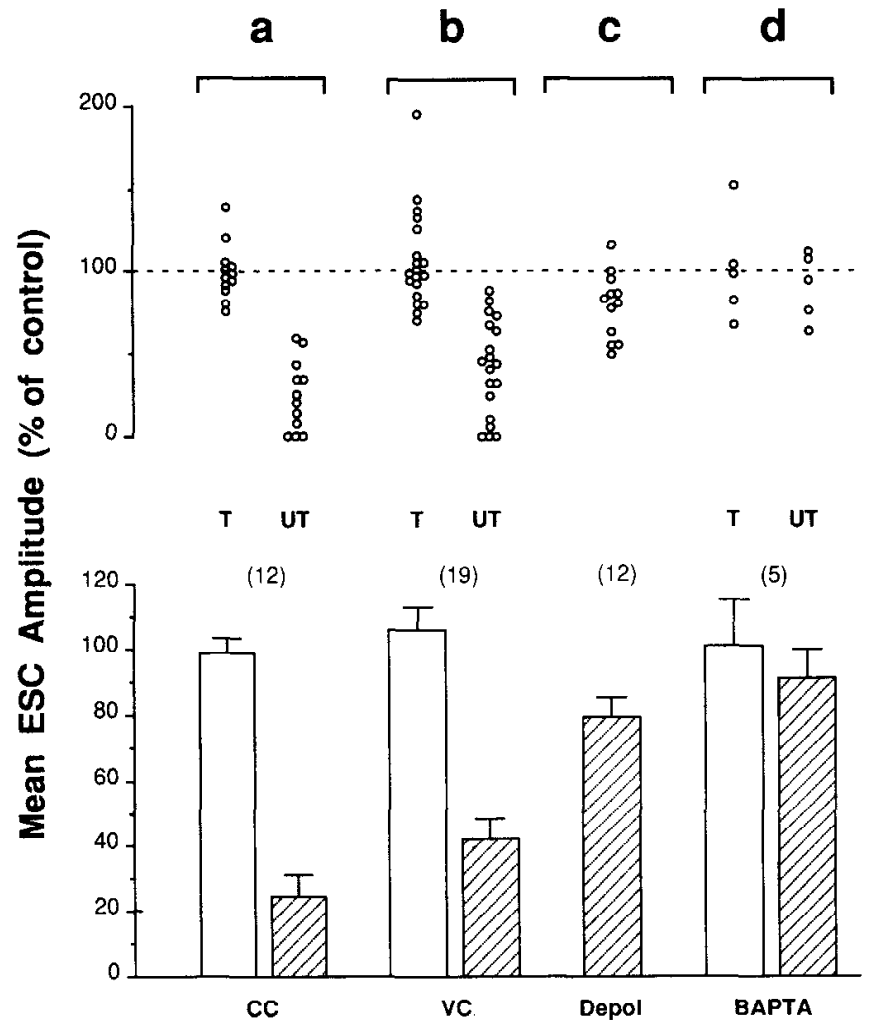

Figure 3. Summary of results from four groups of experiments, presented as scatter plots (upper graphs) and as histograms (lower graphs). Each data point in the scatter plot depicts the result from a single experiment, and lateral displacement of the points is for clarity of presentation only. The mean ESC amplitude within the first 10 min following the tetanus was normalized to the pretetanus mean ESC amplitude for the tetanized neuron $(T)$ and the untetanized neuron $(U T)$ in $a, b$, and $d$, and for single innervation in $c$. For corresponding histograms below, synaptic response was calculated as the percentage of control of the mean ESC amplitude following the tetanus for each neuron shown in the scatter plot above and averaged to yield mean percentage \pm SEM. The numbers associated with the data refer to the total number of experiments performed. $a$, One of the two neurons innervating the myocyte was tetanized by 100 pulses at $2 \mathrm{~Hz}$ while the myocyte recording was switched to current-clamp $(C C)$ condition. $b$, Same as in $a$ except the tetanus was applied under voltage-clamp $(V C)$ condition. $c$, Repetitive suprathreshold depolarization was applied to singly innervated myocytes by injections of current pulses ( 100 pulses at $2 \mathrm{~Hz}$ ) through the whole-cell recording pipette $(2 \mathrm{~Hz}, 50 \mathrm{sec}) . d$, The same as $b$ except that the whole-cell recording pipette contained 5 mM BAPTA and $2 \mathrm{mM} \mathrm{Ca}^{2+}$. The hatched bars refer to the UT inputs and the open bars refer to the $\mathrm{T}$ inputs.

the cytoplasm (Adler et al., 1991), the estimated cytosolic $\mathrm{Ca}^{2+}$ concentration after BAPTA application was about $100 \mathrm{~nm}$. The normalized mean ESC amplitude was determined for each synaptic input after tetanic stimulation ( 100 pulse, $2 \mathrm{~Hz}$ ) was preferentially applied to one presynaptic neuron. The normalized mean ESC amplitudes were $101 \pm 14 \%$ and $91.2 \pm 9.1 \%( \pm$ SEM, $n=5$ ) for the tetanized and untetanized inputs, respectively (Fig. $3 d$ ), which were not significantly different ( $p>0.5, t$ test). In comparison to heterosynaptic suppression under normal conditions (without BAPTA), the percentage reduction in mean ESC amplitude with BAPTA was significantly smaller $(p<0.05$, $t$ test). Thus, heterosynaptic suppression was significantly reduced when a rise of myocyte $\mathrm{Ca}^{2}+$ level was reduced. This result suggests that postsynaptic $\mathrm{Ca}^{2+}$ elevation may be responsible for the induction of heterosynaptic suppression.

\section{Persistence and accumulation of heterosynaptic suppression}

To better understand the long-term functional implication of heterosynaptic suppression, we have studicd the persistence and the accumulation of heterosynaptic suppression. Figure 4 depicts the results from nine experiments in which the recording was made for at least 20 min after one episode of tetanic stimulation (100 pulses at $2 \mathrm{~Hz}$ ) was applied to one of two coinnervating neurons. One case showed complete recovery and three other cases showed partial recovery after initial suppression, while the remaining five cases showed no apparent recovery over the period of observation. No obvious difference in the persistence of suppression was found for cases that employed voltage-clamp versus current-clamp conditions during tetanus.

Since heterosynaptic suppression appears to persist after a brief tetanus, we examined whether repeated episodes of tetanic stimulation of one synaptic input can lead to accumulation of the suppressive effect. In all 4 cases using two repeated episodes of preferential tetanic stimulation $(2 \mathrm{~Hz}, 100$ pulses each), the synaptic efficacy of the untetanized inputs was found to be further suppressed after the second episode of tetanus, and two of the four cases showed total suppression. In contrast, for all single-tetanus experiments ( 31 cases), only six cases showed total suppression after the tetanus. Figure 5 depicts average synaptic responses for all four cases of two-tetani experiments.

\section{Dependence on the number and frequency of tetanic pulses}

To further investigate the amount of tetanic stimulation required to induce detectable heterosynaptic suppression, the total number of tetanic pulses applied to one of the presynaptic neurons was varied from 10 to 100 , while the frequency of stimulation was kept at $2-2.5 \mathrm{~Hz}$. The mean ESC amplitude elicited by the untetanized input within a $10 \mathrm{~min}$ period before and after the tetanus was determined. As shown in Figure 6, significant suppression was found after 20 pulses of stimulation. In those experiments using 10 pulses of tetanic stimulation, three out of six cases showed small but significant suppression.

The following observations suggest that the dominant factor in the induction of heterosynaptic suppression is the total number of pulses applied, rather than the frequency of stimulation. During test stimulation at a low frequency of $0.05 \mathrm{~Hz}$, we observed that in a minority of cells ESCs elicited by both coinnervating neurons showed gradual decline in their amplitudes, suggesting that the test stimuli may have induced synaptic depression. This synaptic depression appears to be due to heterosynaptic suppression, since the synaptic response at one depressed input showed either complete or partial recovery when the test stimulation applied to the other input was terminated. Two extreme examples of suppression by the test stimuli are illustrated in Figure 7. Furthermore, in a series of experiments we have systematically varied the frequency of 100 tetanic pulses from 0.5 to $5 \mathrm{~Hz}$. The effect of heterosynaptic suppression was found to be similar for the four different stimulation frequencies (Fig. 8), suggesting heterosynaptic suppression is independent of the tetanus frequency over the range of $0.5-5 \mathrm{~Hz}$.

The role of spontaneous synaptic activity in synaptic competition

We have previously observed that at some devcloping neuromuscular synapses in these Xenopus cultures, a few presynaptic nerve impulses could result in "bursting" spontaneous release of $\mathrm{ACh}$, as shown by a marked increase in the frequency of SSCs 


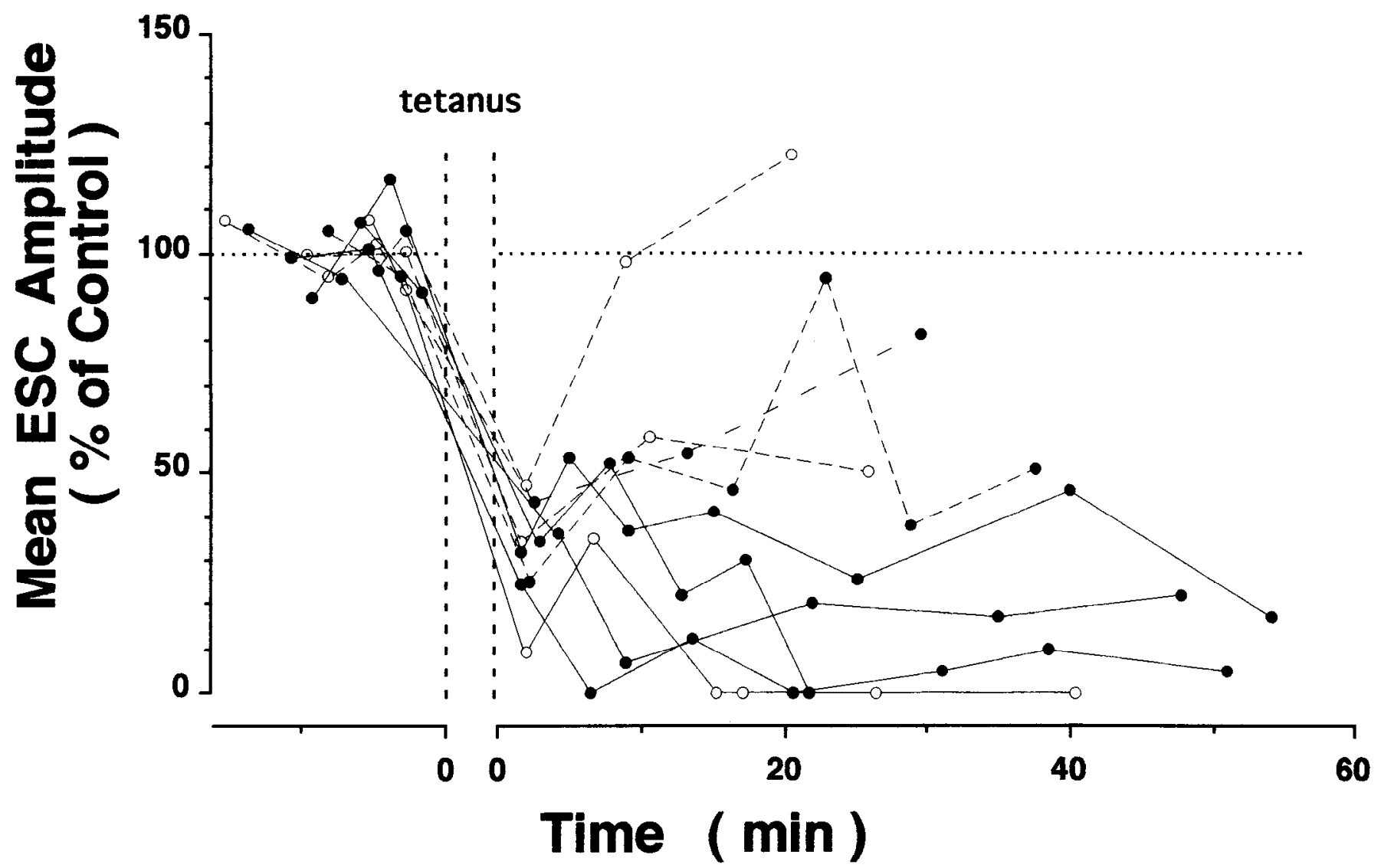

Figure 4. The time course of the heterosynaptic suppression. Tetanic stimulation (100 pulses at $2 \mathrm{~Hz}$ ) was applied to one of the two neurons coinnervating the same myocyte. Each data point represents the mean amplitude of ESC.s elicited by five or six test stimuli applied to the untetanized neuron, normalized to the pretetanus mean ESC amplitude. Data of all cases for which the posttetanus recording period lasted for 20 min or longer are presented. Partial or full recovery of synaptic response after heterosynaptic suppression in four experiments (dashed lines). Results of five other experiments showed no recovery (solid lines). Open and solid symbols refer to experiments employing current-clamp and voltage-clamp conditions during tetanus, respectively.

(Lo et al., 1991). In the present study, this impulse-induced bursting of ACh release was observed in approximately $20 \%$ of the cases (see also Lo et al., 1991) during the low-frequency test stimulation. Interestingly, we found that such spontaneous ac-

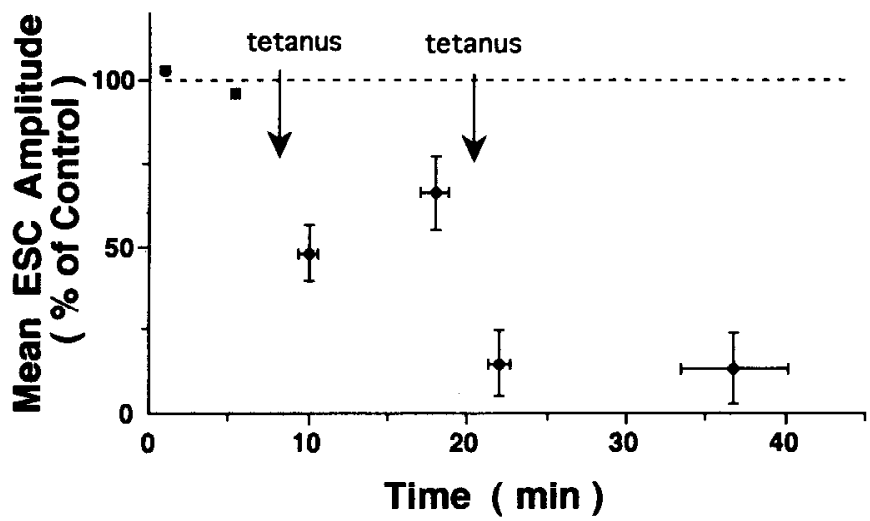

Figure 5. Accumulation of heterosynaptic suppression by repeated episodes of tetanic stimulation. Data from four experiments in which two episodes of tetanic stimulation ( 100 pulses, $2 \mathrm{~Hz}$, at times marked by the arrows) were applied to one of the coinnervating neurons while the myocyte was under voltage-clamp condition. Data points refer to the mean ESC amplitude elicited by the untetanized neuron, normalized to the pretetanus mean ESC amplitude prior to averaging. Error bars refer to SEM. tivity was apparently effective in causing heterosynaptic suppression. In cases where test stimuli induced bursting spontaneous release, the mean amplitude of ESCs elicited by the unstimulated neuron was reduced to $81 \pm 6.2 \%$ ( \pm SEM, $n=$

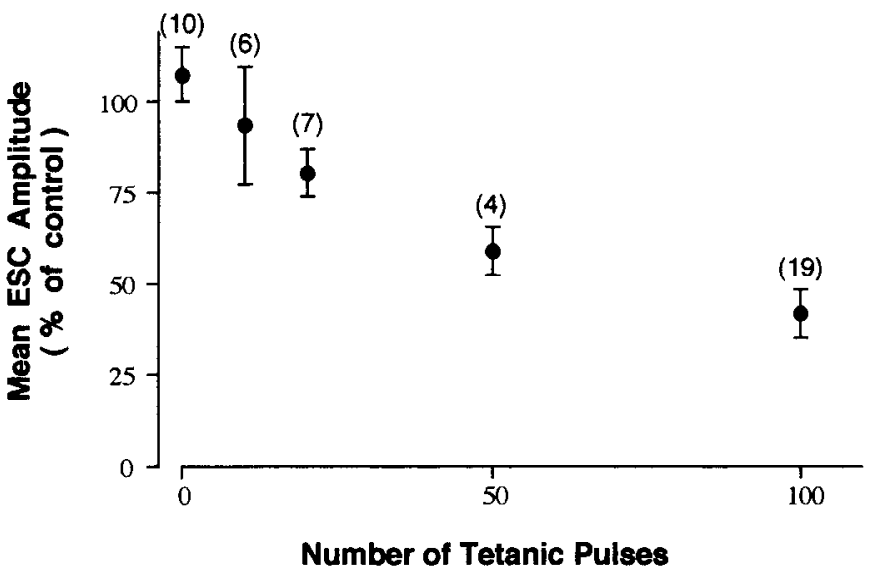

Figure 6. Dependence of heterosynaptic suppression on the number of stimulation pulses in the tetanus. The myocytes were voltage clamped during the tetanus and the frequency of the tetanus was set at $2 \mathrm{~Hz}$ while the number of stimuli varied from 10 to 100 . The mean amplitude of ESCs elicited by the untetanized neuron assayed within the first $10 \mathrm{~min}$ following the tetanus was normalized to pretetanus mean ESC amplitude prior to averaging. Error bars refer to SEM, and the numbers refer to the number of cells examined. 


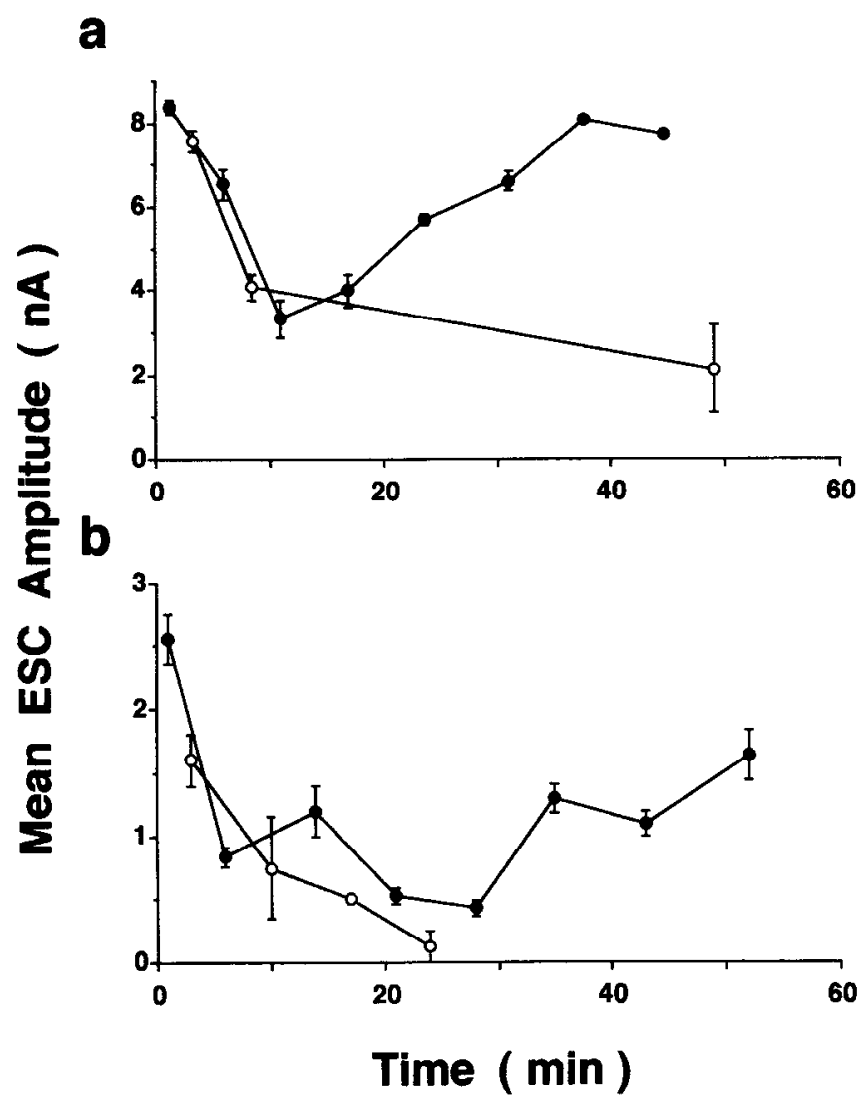

Figure 7. Slow synaptic depression induced by low-frequency test stimuli. Alternating tests of synaptic efficacy were performed by presynaptic suprathreshold stimulation for five or six pulses (at $0.05 \mathrm{~Hz}$ ) for every $5 \mathrm{~min}$. The mean ESC amplitudes elicited by two innervating neurons were depicted separately by open and solid circles. Error bars refer to SEM. $a$, Test stimulation on one neuron (open circles) was terminated after two tests, 10 min after the onset of the experiment. The ESCs elicited by the other neuron (solid circles) recovered to the original level after $30 \mathrm{~min}$, while that of the untested neuron showed further decline. $b$, Similar experiment as in $a$ except the test stimulation of one neuron (open circles) was terminated 25 min after the onset of the experiment. Partial recovery of ESCs was observed for the other neuron.

7) of the prebursting values ( $p<0.05, t$ test). Thus, spontaneous synaptic activity could contributc to synaptic competition when bursting ACh release is triggered.

\section{Presynaptic or postsynaptic modulation?}

The reduction of synaptic efficacy due to heterosynaptic stimulation could result from a postsynaptic reduction of $\mathrm{ACh}$ receptor density or sensitivity, a reduction of the presynaptic $\mathrm{ACh}$ release, a change in the synaptic morphology, or a combination of these factors. Two analyses of the data suggest that a reduction of presynaptic ACh release is likely to be involved. First, we measured the amplitude of SSCs before and after the induction of heterosynaptic suppression. No significant reduction in the mean amplitude of SSCs was observed. The mean amplitude was $98.4 \pm 8.1 \%( \pm \mathrm{SEM}, n=5)$ of the control values prior to the tetanus, and the amplitude distribution of SSCs, as depicted by the cumulative frequency of events at various amplitudes (Fig. 9a), showed no significant change (at a 0.05 level, Kolmogorov-Smirnov test). If suppression involves postsynaptic reduction of $\mathrm{ACh}$ receptor density/sensitivity, we would expect a corresponding reduction in the mean amplitude of the SSCs

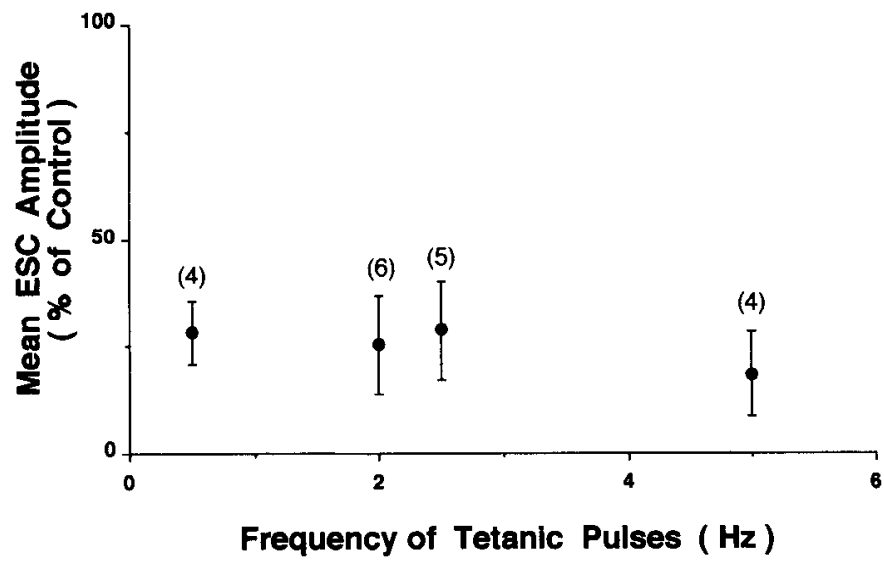

Figure 8. Dependence of heterosynaptic suppression on the frequency of tetanic stimulation. Tetanic stimuli of 100 pulses were applied under myocyte voltage-clamp condition to the stronger presynaptic neurons at either $0.5,2,2.5$, or $5 \mathrm{~Hz}$. The mean ESC amplitudes were determined as in Figure 6. Error bars refer to SEM, and the numbers refer to the number of cells examined.

due to the suppressed synapse and a significant change in their amplitude distribution. However, since we cannot distinguish the population of the spontaneous events originated from the tetanized versus untetanized input, the possibility exists that some reduction as well as elevation of SSC amplitudes had occurred for subpopulations of SSCs and had canceled cach other's effect. The frequency of SSCs recorded in the myocyte showed a significant increase after heterosynaptic suppression (148.8 $\pm 12.6 \%$ of control, $\pm \mathrm{SEM}, n=5$ ). However, this may result from the potentiation of spontaneous $\mathrm{ACh}$ secretion in the tetanized input, a phenomenon previously described (Lo et al., 1991). Whether there was reduction of SSC frequency in the suppressed input cannot be resolved by the present method.

In the second analysis, we measured the coefficient of variation (CV) of the amplitude of ESCs, which is defined as the ratio of $v^{1 / 2} / m$, where $m$ and $v$ are the mean ESC amplitude and its variance, respectively. Assuming that the synaptic transmission in this culture is quantal in nature and that variation of the ESC amplitude can be described by binomial distribution modified to account for the variation of the quantal size, the ratio of $v / m^{2}\left(\mathrm{CV}^{2}\right)$ is a quantity independent of the quantal size and remains constant if changes in synaptic efficacy are due solely to a change in quantal size, such as would result from a postsynaptic change in $\mathrm{ACh}$ sensitivity. In the latter situation, the ratio of $\mathrm{CV}$ squared before and after synaptic modification should be close to 1 . In Figure $9 b$, we plotted the ratio of $\mathrm{v} / \mathrm{m}^{2}$ for a number of cases of heterosynaptic suppression where a relatively large number of ESC events had been recorded immediately before and after application of tetanus. The data all fell below 1 (dashed line) for varying degrees of synaptic suppression, which was defined as the ratio of mean ESC amplitude after tetanus to that before the tetanus. The data clearly indicate a substantial increase in amplitude fluctuation after suppression, suggesting that postsynaptic changes cannot be the only cause of observed synaptic suppression, and that it is likely that presynaptic reduction of $\mathrm{ACh}$ release is involved.

\section{Correlation of heterosynaptic suppression with synapse efficacy}

Throughout this study we found that the degree of heterosynaptic suppression varied greatly (see Fig. 3). Although most of the cases studied showed significant suppression after applica- 
tion of the standard tetanus ( 100 pulses at $2-5 \mathrm{~Hz}$ ) to the heterosynaptic input, some cases showed little or no change. To understand the cause of this variability, we have examined the correlation between the extent of suppression and the synaptic efficacy of both inputs prior to the tetanus. As shown in Figure $10 a$, we found a positive correlation between the extent of suppression and the ratio of synaptic efficacy between the tetanized and untetanized inputs (coefficient $=0.51$ ). In other words, the effect of the heterosynaptic suppression increased with the disparity in the synaptic efficacy of the two inputs. We also examined the correlation between the suppression effect and the initial synaptic efficacy of the untetanized input. A negative correlation was observed (coefficient $=-0.47$ ) (Fig. 10b), suggesting that weaker inputs were more susceptible to heterosynaptic suppression.

\section{Discussion}

Activity-dependent synaptic competition between coinnervating nerve terminals on the same postsynaptic cells is believed to be important for determining the mature pattern of innervation in both CNS and PNS (Purves and Lichtman, 1985; Goodman and Shatz, 1993). The critical role of activity in the elimination of polyneuronal innervation of skeletal muscles during development is well-established (Betz et al., 1990; Van Essen et al., 1990), although the mechanisms by which the activity affects the neuronal connection and the nerve terminals compete for their survival remain unknown. One approach to elucidate the cellular basis of synaptic competition is the use of culture systems, where the pre- and postsynaptic cells are more accessible to experimental manipulation. Using cultures of chick ciliary ganglia explants and muscle cells, Magchielse and Meeter (1986) showed that phasic stimulation of one ganglion explant resulted in selective survival of the neuromuscular synapses made by stimulated ganglion and elimination of synapses made by nonstimulated ganglion. In cultures of mouse muscle cells and superior cervical ganglion or ventral spinal cord neurons, electrical stimulation of presynaptic axons caused a significant increase in functional elimination of synapses in the culture, as compared to unstimulated cultures. However, the extent of elimination of activated versus unactivated synapses in the stimulated culture was similar, suggesting the activity did not confer any selective advantage in the process of synapse elimination (Nelson et al., 1993). On the other hand, in Xenopus nerve-muscle cultures, we have shown that preferential tetanic stimulation of one presynaptic spinal neuron resulted in heterosynaptic suppression of synaptic inputs made by untetanized neurons innervating the same postsynaptic cell, while the tetanized inputs remained unchanged or potentiated (Lo and Poo, 1991). The present study provides further characterization of this phenomenon of heterosynaptic suppression in Xenopus cultures, and resolves some of the outstanding questions concerning the involvement of postsynaptic depolarization, the persistence of the suppression, the pattern of tetanus required to induce suppression, and possible cellular mechanisms involved in the induction and expression of synaptic suppression.

The first set of experiments showed that heterosynaptic suppression could be induced by applying tetanic stimulation under either voltage- or current-clamp conditions, with a slightly high$\mathrm{cr}$ extent of suppression for the current-clamp condition. $\mathrm{Al}$ though postsynaptic $\mathrm{ACh}$ receptors were activated in both cases, the muscle cells were allowed to be repetitively depolarized under the latter condition. One consequence of such depolar-
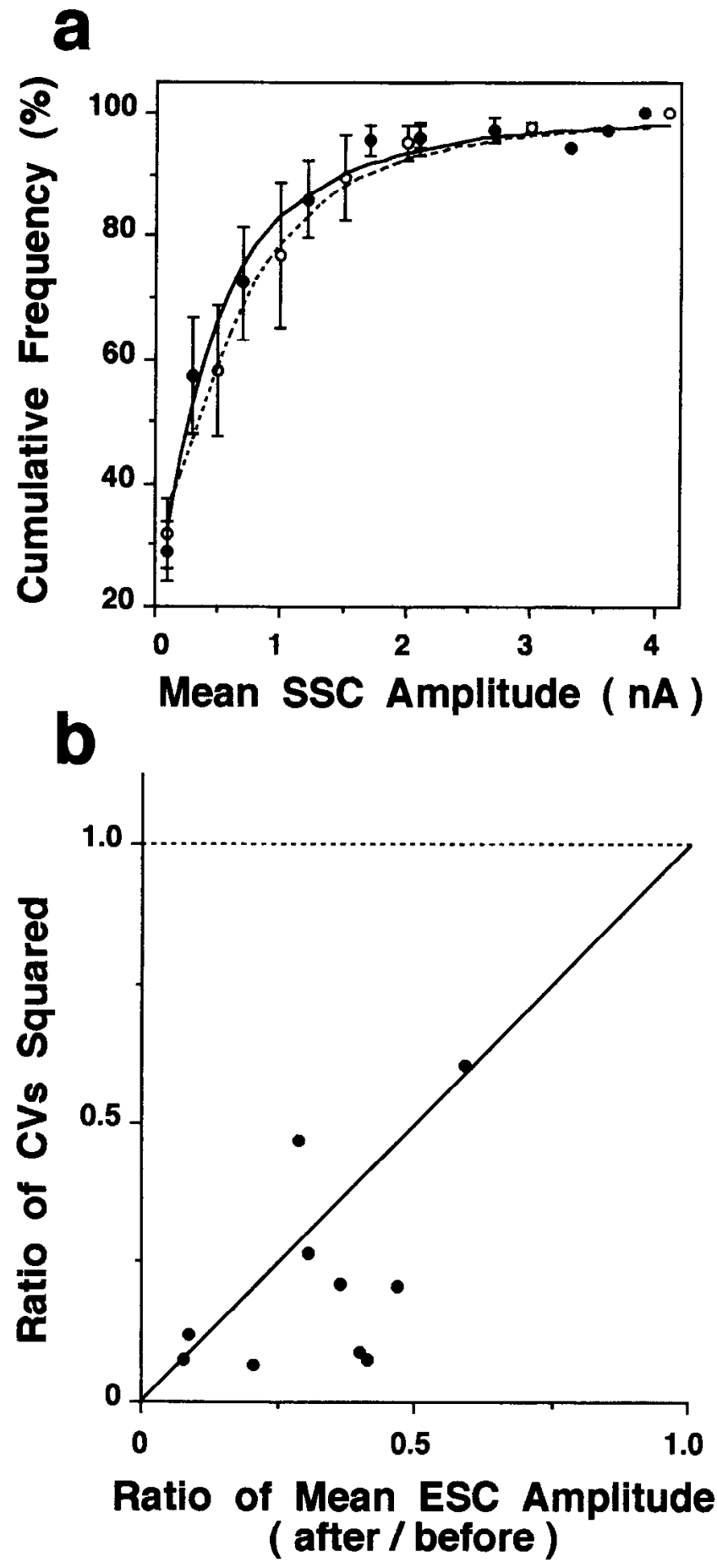

Figure 9. a Amplitude distribution of spontaneous synaptic currents ( $S S C s$ ). The cumulative frequency refers to the percentage of total events exhibiting amplitudes below the corresponding amplitude value. Average amplitude distributions before and after induction of heterosynaptic suppression by tetanic stimulation from five experiments under VC condition are depicted by the dashed line (open circles) and solid line (solid circles), respcctivcly. For clarity, only sclcctivc data points are displayed. Error bars refer to SEM. $b$, Analysis of the ESC amplitude fluctuation for the suppressed synapses. The ratio of coefficients of variation $(C V)$ squared before and after suppression $\left(C V_{h}^{2} / C V_{a}^{2}\right)$ was plotted versus the extent of suppression, as represented by the mean ESC amplitude after tetanus/mean ESC amplitude before tetanus. Each point represents data from one experiment. Dashed line represents theoretical prediction for pure postsynaptic changes in synaptic responses, and the solid diagonal line represents the prediction for pure presynaptic changes in transmitter secretion. 

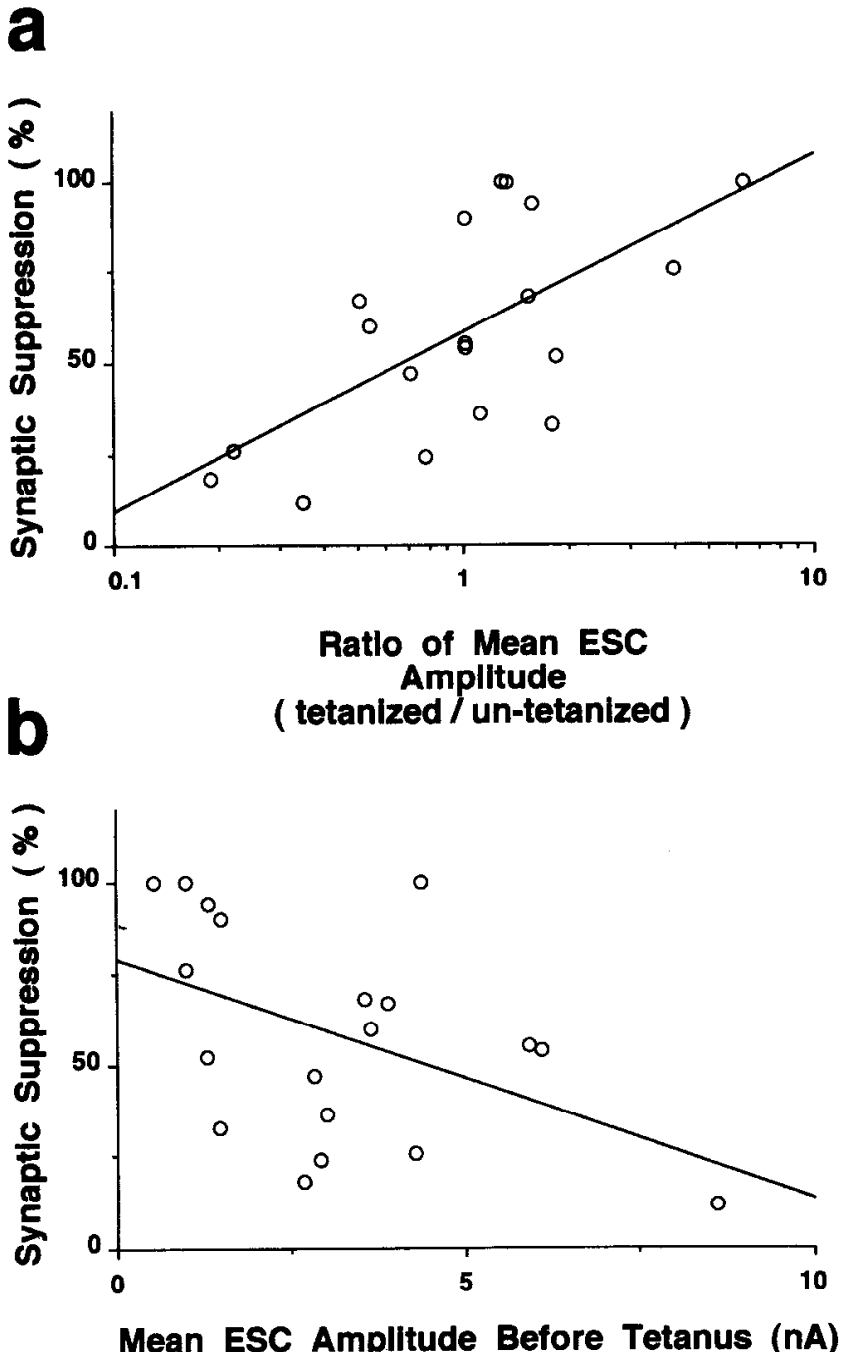

Figure 10. Correlation of the extent of the heterosynaptic suppression with the initial synaptic efficacy of the synapse. Each open circle represents an individual experiment. $a$, Synaptic suppression (\%) is defined as the percentage of reduction in the mean ESC amplitude of the untetanized synapse after the tetanus, and is plotted against the ratio of synaptic efficacy, that is, the mean ESC amplitude of the tetanized synapse/the mean ESC amplitude of the untetanized synapse. Solid line represents correlation coefficient of 0.51 . The positive correlation indicates that the extent of heterosynaptic suppression increases with the relative strength of the tetanized synapse. $b$, The percentage suppression is plotted against the mcan ESC amplitude of the untetanized synapse before tetanic stimulation. Solid line represents correlation coefficient of -0.47 .

ization may be the opening of voltage-dependent $\mathrm{Ca}^{2+}$ channels in the myocyte plasma membrane and subsequent increase of the $\mathrm{Ca}^{2+}$ influx into the myocyte. Although a substantial amount of $\mathrm{Ca}^{2+}$ influx is known to occur through activated $\mathrm{ACh}$ channels (Decker and Dani, 1990), independent of the membrane depolarization, additional $\mathrm{Ca}^{2+}$ influx through voltage-dependent $\mathrm{Ca}^{2+}$ channels may help to elevate the total amount of $\mathrm{Ca}^{2+}$ influx during the tetanus. The importance of postsynaptic $\mathrm{Ca}^{2+}$ influx in heterosynaptic suppression is shown by the finding that buffering the $\mathrm{Ca}^{2+}$ concentration in the myocyte to a low level using BAPTA significantly reduced the suppression. This result is consistent with the previous finding that $\mathrm{Ca}^{2+}$ elevation in the myocyte is required for the synaptic depression induced by repetitive activation of myocyte $\mathrm{ACh}$ receptors by iontophoresis on a singly innervated Xenopus myocyte (Dan and Poo, 1992). Furthermore, postsynaptic depolarizations induced by direct current injections alone are sufficient to produce synaptic depression, especially after prolonged duration of repetitive depolarizations (Lo et al., 1994). In the latter study, synaptic depression was also reduced by buffering the cytosolic $\mathrm{Ca}^{2+}$ of the myocyte at a low level. A crucial role of $\mathrm{Ca}^{2+}$ in synaptic competition has also been shown by a study of activity-dependent modulation of synaptogenesis between sensory afferents and spinal cord neurons (Nelson et al., 1990). In the latter case, $\mathrm{Ca}^{2+}$ influx through postsynaptic glutamate channels and voltagedependent $\mathrm{Ca}^{2+}$ channels may be responsible for synapse stabilization and elimination.

Direct presynaptic interactions between the coinnervating nerve terminals, if it exists at all, does not seem to play a major role in the induction of heterosynaptic suppression, since the suppression was significantly reduced when a rise of postsynaptic $\mathrm{Ca}^{2+}$ was prevented. Does the suppression of synaptic efficacy by heterosynaptic stimulation result from changes in the postsynaptic AChR density/sensitivity, presynaptic ACh secretion, or both? Several results provided indirect evidence that reduced presynaptic ACh secretion contributes to synaptic suppression. The spontaneous synaptic currents observed in the innervated myocytes, although due to both innervating terminals, showed no detectable change in the amplitude distribution following the induction of heterosynaptic suppression. If the suppressed input contributed a substantial number of the recorded spontaneous events, our result would suggest that postsynaptic sensitivity at the suppressed input was not significantly reduced. However, the reduction of SSC amplitude, if it exists at all, may be undetectable when the suppressed input contributes only a small fraction of the SSC events. The result of fluctuation analysis on the variation of ESC amplitudes is inconsistent with a purely postsynaptic reduction of $\mathrm{ACh}$ sensitivity as the cause of synaptic suppression. The most likely mechanism of reduced synaptic efficacy is a reduction in the quantal content, that is, the number of $\mathrm{ACh}$ quanta released by presynaptic action potentials, as a result of either a reduced release probability or reduced number of available quanta, or both. However, our results cannot exclude some elaborate mechanism of postsynaptic changes. For example, if the postsynaptic ACh receptors exist as clusters and each quantal response is restricted to activation of a single cluster, selective switching of postsynaptic receptor clusters from "on" to "off" states could leave the response to individual quanta unchanged but reduce the total evoked response. In addition, some nerve terminal arbors could have retracted from the myocyte surface, leading to reduced ACh response without changes in the presynaptic release mechanism or postsynaptic sensitivity.

We have observed that under the standard tetanus ( 100 pulses at $2 \mathrm{~Hz}$ ), the extent of heterosynaptic suppression varied greatly (Fig. 3). There are several possible causes of this variability. First, there is heterogeneity of neuronal types in these cultures (Bixby and Spitzer, 1984). Among the cholinergic neurons used in these experiments, there may be intrinsic cell-type differences in their susceptibility to synaptic suppression. Second, the ability to induce heterosynaptic suppression may be related to the strength of the tetanized inputs as compared to the untetanized ones. Third, the status of synapse maturation prior to the tetanus may be related to the susceptibility of the synapse to heterosynaptic suppression. The latter two possibilities were supported by the positive correlation between the ratio of the synaptic 
efficacy (tetanized input/untetanized input) and the extent of heterosynaptic suppression, and by the negative correlation between the efficacy of untetanized inputs prior to the tetanus and the extent of heterosynaptic suppression (Fig. 10). Fourth, the extent of heterosynaptic suppression may depend on the proximity of the innervation sites between two neurons. We have previously found that, for dually innervated spindle-shaped myocytes, no heterosynaptic suppression could be induced by preferential tetanic stimulation when the innervation sites were separated by a distance larger than $50 \mu \mathrm{m}$ on spindle shaped myocytes (Lo and Poo, 1991). Although the innervating sites on the spherical myocytes used in the present study are confined within a distance set by the myocyte diameter (mean of $35 \mu \mathrm{m}$ ), some variation in the proximity between the synapses is expected. Finally, the spontaneous secretion of $\mathrm{ACh}$ from each neuron might also contribute in part to the synaptic competition. This contribution cannot be estimated quantitatively since the present recording method cannot identify from which presynaptic neuron a particular spontaneous event in the myocyte originated. Consistent with the potential role of spontaneous synaptic events, we have found that bursting spontaneous $\mathrm{ACh}$ secretion triggered by intense (sometimes damaging) electrical stimulation, which was severalfold larger in strength than that used in the standard heterosynaptic stimulation, to one presynaptic neuron often resulted in detectable suppression of the synaptic response elicited by the unstimulated neuron.

Functional intcractions between coinnervating nerve terminals have been observed in neonatal rat lumbrical muscle (Betz et al., 1989). In this study, a single conditioning stimulation of one of the coinnervating nerves often reduced the response to test stimulation of the other nerve. Interestingly, this heterosynaptic inhibition was observed even when the muscle membrane was voltage clamped and blocking the $\mathrm{ACh}$ receptors with curare or $\alpha$-bungarotoxin reduced the inhibition. These results suggest the involvement of $\mathrm{ACh}$ receptor activation and perhaps postsynaptic $\mathrm{Ca}^{2+}$ influx in the induction of heterosynaptic inhibition. Furthermore, the inhibition was found to be maximal when the conditioning test interval was short and decayed over a period of $50-100 \mathrm{msec}$ after the conditioning stimulus. It is unknown whether repetitive conditioning stimuli similar to those used in the present study could induce persistent inhibition.

Finally, we note that the persistence of heterosynaptic suppression and the cumulative effect of successive episodes of tetani suggest that long-lasting functional suppression of the synapse can be induced by preferential presynaptic activity. Such functional suppression may be a prelude to more permanent structural changes, for example, retraction of the suppressed nerve terminal, that underlie activity-dependent synapse elimination.

\section{References}

Adler EM, Augustine GJ, Duffy SN, Charlton MP (1991) Alien intercellular calcium chelators attenuate neurotransmitter release at the squid giant synapse. J Neurosci 11:1496-1507.

Anderson MJ, Cohen MW, Zorychta E (1977) Effects of innervation on the distribution of acetylcholine receptors on cultured muscle cells. J Physiol (Lond) 268:731-756.

Bekkers JM, Stevens CF (1990) Presynaptic mechanism for long-term potentiation in the hippocampus. Nature 346:724-729.

Betz WJ, Chua M, Ridge RMAP (1989) Inhibitory interactions be- tween motoneurone terminals in neonatal rat lumbrical muscle. $J$ Physiol (Lond) 418:25-51.

Betz WJ, Ribchester, RR, Ridge RMAP (1990) Competitive mechanisms underlying synapse elimination in the lumbrical muscle of the rat. J Neurobiol 21:1-17.

Bixby JL, Spitzer NC (1984) The appearance and development of neurotransmitter sensitivity embryonic spinal neurons in vitro. $\mathrm{J}$ Physiol (Lond) 353:143-155.

Buchanan J, Sun Y, Poo M (1989) Studies of nerve-muscle interactions in Xenopus cell culture: fine structure of early functional contacts. $\mathrm{J}$ Neurosci 9:1540-1554.

Constantine-Paton M, Cline HT, Debski E (1990) Patterned activity, synaptic convergence, and the NMDA receptor in developing visual pathways. Annu Rev Neurosci 3:129-154.

Dan Y, Poo M-m (1992) Hebbian depression of isolated neuromuscular synapses in vitro. Science 256:1570-1573.

Decker ER, Dani JA (1990) Calcium permeability of the nicotinic acetylcholine receptor: the single-channel calcium influx is significant. J Neurosci 10:3413-3420.

Evers J, Laser M, Sun Y, Xie Z, Poo M-m (1989) Studies of nervemuscle interactions in Xenopus cell culture: analysis of early synaptic currents. J Neurosci 9:1523-1539.

Goodman CS, Shatz CJ (1993) Developmental mechanisms that generate precise patterns of neuronal connectivity. Neuron [Suppl] 10: 77-98.

Hamill OP, Marty A, Neher E, Sakmann B, Sigworth FJ (1981) Improved patch-clamp techniques for high-resolution current recording from cell and cell-free membrane patches. Pfluegers Arch 391:85100 .

Kullberg R, Lentz T, Cohen MW (1977) Development of myotomal neuromuscular junction in Xenopus laevis: an electrophysiological and fine-structural study. Dev Biol 60:101-129.

Lo YJ, Poo M-m (1991) Activily-dependent synaptic competition in vitro: heterosynaptic suppression of developing synapses. Science 254: 1019-1022.

Lo YJ, Wang T, Poo M-m (1991) Repetitive impulse activity potentiates spontaneous acetylcholine secretion at developing neuromuscular synapses. J Physiol (Paris) 85:71-78.

Lo YJ, Lin Y-c, Sanes DH, Poo M-m (1994) Depression of developing neuromuscular synapses induced by repetitive postsynaptic depolarizations. I Neurosci 14:4694-4704.

Magchielse T, Meeter E (1986) The effect of neuronal activity on the competitive elimination of neuromuscular junctions in tissue culture. Dev Brain Res 25:211-220.

Malinow R, Tsien RW (1990) Presynaptic enhancement shown by whole-cell recordings of long-term potentiation in hippocampal slices. Nature 346:177-180.

Nelson PG, Fields RD, Yu C, Neale EA (1990) Mechanisms involved in activity-dependent synapse formation in mammalian central nervous system cell cultures. J Neurobiol 21:138-156.

Nelson PG, Fields RD, Yu C, Liu Y (1993) Synapse elimination from the mouse neuromuscular junction in vitro: a non-Hebbian activitydependent process. J Neurobiol 24:1517-1530.

Nieuwkoop PD, Faber J (1967) Normal table of Xenopus laevis, 2d ed. Amsterdam: North Holland.

Purves D, Lichtman JW (1985) Principles of neural development, pp 289-312. Sunderland, MA: Sinauer.

Shatz CL (1990) Impulse activity and the patterning of connections during CNS development. Neuron 5:745-756.

Spitzer NC, Lamborghini JC (1976) The development of the action potential mechanism of amphibian neurons isolated in culture. Proc Natl Acad Sci USA 73:1641-1645.

Sun Y, Poo M-m (1987) Evoked release of acetylcholine from growing embryonic neuron. Proc Natl Acad Sci USA 84:2540-2544.

Tabti N, Poo M-m (1991) Culturing spinal cord neurons and muscle cells from Xenopus embryos. In: Culturing nerve cells (Banker $\mathrm{G}$, Goslin K, eds), pp 137-154. Cambridge, MA: MIT Press.

Van Essen DC, Gordon H, Soha JM, Fraser SE (1990) Synaptic dynamics at the neuromuscular junction: mechanisms and models. J Neurobiol 21:223-249. 